\title{
Effective Rituximab Treatment in Patients with Neuromyelitis Optica Spectrum Disorders Compared with Azathioprine and Mycophenolate
}

\author{
Yang Yang $\cdot$ Lifeng Chen $\cdot$ Lei Wu $\cdot$ Jiarui Yao $\cdot$ Na Wang $\cdot$ \\ Xiaoqing $\mathrm{Su} \cdot$ Dongmei Li · Lina Han · Weiping Wu • \\ Dehui Huang $\cdot$ Tianyu Jiang $\cdot$ Zhenfu Wang (D)
}

Received: September 5, 2021 / Accepted: October 27, 2021 / Published online: November 19, 2021

(c) The Author(s) 2021

\section{ABSTRACT}

Introduction: As an autoimmune central nervous system disease characterized by inflammation and demyelination, neuromyelitis

Yang Yang and Lifeng Chen contributed equally to this work.

Supplementary Information The online version contains supplementary material available at https:// doi.org/10.1007/s40120-021-00298-5.

Y. Yang · J. Yao - X. Su - D. Li · L. Han · W. Wu . Z. Wang $(\bowtie)$

Department of Neurology, The Second Medical Center and National Clinical Research Center for Geriatric Diseases, Chinese PLA General Hospital, No.28 Fuxing Road, Haidian District, Beijing, China e-mail: wuwp301@163.com

\section{Chen}

Department of Neurosurgery, The First Medical Center, Chinese PLA General Hospital, No.28

Fuxing Road, Haidian District, Beijing, China

L. Wu · D. Huang $(\bowtie)$

Department of Neurology, The First Medical Center, Chinese PLA General Hospital, No.28 Fuxing Road, Haidian District, Beijing, China

e-mail: huangdehui@gmail.com

N. Wang $\cdot$ T. Jiang $(\varangle)$

Department of Rehabilitation Medicine, The Second Medical Center and National Clinical Research Center for Geriatric Diseases, Chinese PLA General Hospital, No.28 Fuxing Road, Haidian District, Beijing, China

e-mail: jty301@126.com optica (NMO) has been extensively investigated. A specific antigenic target, astrocytic water channel aquaporin-4 (AQP4) has already been identified, and it can be recognized explicitly by the autoantibody marker NMOIgG. Along with the immune attacks, clinical disabilities would gradually accumulate. As there has been no validated and well-recognized therapy for NMO till now, preventing and postponing attack using immunosuppressive therapies is the primary treatment option.

Methods: In the current retrospective study, the effect of immunosuppressive agents was investigated through a long-term follow-up. To assess the long-term effectiveness and safety of rituximab (RTX), azathioprine (AZA), and mycophenolate mofetil (MMF) therapies, all 129 patients with NMO spectrum disorders (NMOSD) who received at least one of these treatments were studied, including 55 seropositive for $\mathrm{AQP} 4-\mathrm{Ab}$ and 74 seronegative for AQP4-Ab.

Results: The median post-treatment annualized relapse rate (ARR) was lower than the pretreatment rates in all $\mathrm{AQP} 4^{+} \mathrm{Ab}$ groups (from 1.0 to 0.7 in RTX, from 0.8 to 0.3 in AZA, and from 0.85 to 0.35 in MMF). Meanwhile, the ARR also decreased in all $\mathrm{AQP}^{-} \mathrm{Ab}$ groups (from 0.3 to 0.2 in RTX, from 0.9 to 0.5 in AZA, and from 0.9 to 0.4 in MMF). Disability condition improved in the Expanded Disability Status Scale (EDSS) in all $\mathrm{AQP} 4^{+} \mathrm{Ab}$ groups (from 4.0 to 2.75 in RTX, from 3.5 to 2.5 in AZA, and from 3.0 to 2.0 in 
$\mathrm{MMF}$ ) and in all $\mathrm{AQP} 4^{-} \mathrm{Ab}$ groups (from 3.0 to 2.5 in RTX, from 3.0 to 2.5 in AZA, and from 3.5 to 2.0 in MMF). There was no statistically significant difference between the post-treatment and pre-treatment changes of EDSS and ARR in the RTX, AZA, and MMF groups $(P>0.05)$. However, according to Kaplan-Meier survival analysis, RTX-treated patients were more likely to be relapse-free after long-term follow-up than those who received AZA or MMF therapy. Meanwhile, adverse effects were noted in three out of 23 patients with RTX treatment, five of 32 with AZA treatment, and three of 21 with MMF treatment. No serious adverse events were observed in all treatment groups during the study.

Conclusions: RTX, AZA, and MMF therapies efficiently lowered the relapse frequency and disability in both of the AQP4-Ab seropositive or seronegative patients with NMO. Furthermore, low dosage of RTX is recommended for the patients with NMO owing to its long-term effectiveness and safety.

Keywords: Neuromyelitis optica; Aquaporin-4; Rituximab; Azathioprine; Mycophenolate mofetil

\section{Key Summary Points}

Rituximab (RTX), azathioprine (AZA), and mycophenolate mofetil (MMF) are effective, with significant improvements in the Expanded Disability Status Scale (EDSS) and annualized relapse rate (ARR), regardless of the seropositive or seronegative aquaporin- 4 antibody status.

RTX is recommended as the first-line therapy in patients with neuromyelitis optica spectrum disorders rather than AZA and MMF, in view of its effectiveness and safety.

Low dosage of RTX could be recommended for patients with neuromyelitis optica owing to its longterm effectiveness and safety.

\section{INTRODUCTION}

As an idiopathic autoimmune inflammatory demyelinating disease, neuromyelitis optica (NMO) mainly affects the optic nerve and spinal cord of the central nervous system (CNS). NMO is a severe and slow recovery disorder, compared to multiple sclerosis (MS). Identification of astrocytic water channel aquaporin-4 (AQP4) made NMO the first CNS autoimmune inflammatory demyelinating disease that has an identified specific antigenic target. The pathogenesis of $\mathrm{NMO}$ is related to AQP4 specific antibody (AQP4-immunoglobulin $\mathrm{G}$ [IgG]) that differentiates NMO from MS [1, 2]. NMO spectrum disorders (NMOSD) include AQP4-Ab seropositive patients with optic neuritis, longitudinally extensive transverse myelitis, diencephalon, brainstem, and cerebral syndromes. AQP4-Ab seropositivity in NMO or NMOSD is predictive of further relapses $[3,4]$. Fifty percent of patients are wheelchair-dependent, and $62 \%$ are functionally blind without treatment during the first 5 years. Thus, in patients with NMOSD, the primary treatment goal is attack prevention with immunosuppressive therapy or use of a monoclonal antibody such rituximab (RTX) [5]. The most commonly used drugs in China are azathioprine (AZA), mycophenolate mofetil (MMF), and RTX. In recent years, research has confirmed the effectiveness and safety of AZA, MMF, and RTX in the treatment of NMOSD. Moreover, AZA [6] and MMF [7-10] have been widely used, while corticosteroids [11], mitoxantrone [12, 13], cyclophosphamide, and cyclosporin can also be considered. Thus, RTX has been gradually used as first-line choice for NMO treatment, to seek better control of the disease [14-24].

RTX, a chimeric anti-CD20 antibody, was initially used to treat non-Hodgkin lymphoma [25]. The efficiency of RTX in the treatment of $\mathrm{NMO} / \mathrm{NMOSD}$ has been proven, with defined depletion of memory $\mathrm{B}$ cells as a therapeutic target $[17,20]$. However, when used to treat other autoimmune diseases, adverse effects are reported, most frequently including chills, fever, nausea, vomiting, headache, bronchitis, gingivitis, hypotension, thrombocytopenia, 
and neutropenia. Nevertheless, compared with other immunosuppressive agents, AZA, MMF, and RTX are still considered to bear fewer side effects $[7,12,18,20,25]$. However, the treatment response of immunosuppressive agents between aquaporin- 4 antibody positive and negative status in patients with NMOSD, and the long-term effects of immunosuppressive agents in preventing NMO attacks remain controversial.

Though the effectiveness and acceptable safety of treatment with repeated cycles of RTX and long-term treatment with AZA or MMF have been shown by available clinical data, their long-term effectiveness and safety in patients with NMO need to be further determined. Furthermore, taking the high price of RTX and potential adverse effects into consideration, lower dosages of RTX for the treatment of NMOSD have been selected. In this study, differences in the effectiveness and safety associated with lower dosage of RTX $(500 \mathrm{mg}$ infused, then $100 \mathrm{mg}$ if the percentage of $\mathrm{CD}^{+}{ }^{+} \mathrm{B}$ cells reached 1\%) [18] have been compared with AZA ( $2 \mathrm{mg} / \mathrm{kg}$ daily) and MMF (1000 mg daily) treatments assessed in Chinese patients with NMOSD with seropositive and seronegative AQP-4 antibodies in a median 90-month follow-up.

\section{METHODS}

A retrospective review of medical records from the Chinese PLA General Hospital showed that 129 patients with NMOSD (all met the international consensus diagnostic criteria) $[21,26]$ were treated with immunosuppressive drugs and also oral steroids from January 2008 to November 2018. The baseline clinical characteristics of the patients are presented in Table 1.

A total of 129 patients with NMOSD who were previously treated with immunosuppressive medicine and who had concomitant prednisone had received the AQP-4 antibody (AQP4$\mathrm{Ab})$ detection, including 55 patients seropositive for AQP4-Ab and 74 seronegative for AQP4$\mathrm{Ab}$ (Fig. 1). Among the 55 patients in the AQP4$\mathrm{Ab}$ positive group, 14 received low-dose RTX (500 mg infused, then $100 \mathrm{mg}$ if the percentage of $\mathrm{CD} 19^{+} \mathrm{B}$ cells reached $1 \%$ ) (equivalent to $375 \mathrm{mg} / \mathrm{m}^{2}$ by drip infusion every week for 4 weeks; this regimen was based on that for treatment of B cell lymphoma) [15], 13 patients received azathioprine $(2 \mathrm{mg} / \mathrm{kg}$ daily), and eight patients received MMF (1000 mg daily). Every patient remained on the same low-dosage regimen and the patients received treatment as soon as possible, and they underwent long-term follow-up after the drug treatment.

The remaining 20 of 55 records in the AQP4$\mathrm{Ab}$ positive group were excluded (ten patients received Chinese traditional medicine therapy, eight received only a small prednisolone dosage, and two received no other therapy). Among the 74 patients in the AQP4-Ab negative group, nine received low-dose RTX, 19 patients received azathioprine, and 13 patients received MMF. The remaining 33 of 74 records were excluded (17 patients received Chinese traditional medicine, ten received only a small prednisolone dosage, and six received no other therapy) (Fig. 1, Table 1). The quantitative change in anti-AQP4 antibody levels was measured by using a cell-based assay (CBA) [27]. The cutoff level was $50.0 \mathrm{U} / \mathrm{ml}$, and an antibody titer of more than or equal to 1:100 was recognized as a seropositive status. In addition, a flow cytometry analysis was performed in RTX-treated patients before the first infusion as the baseline level and at 1, 3, 6, and 12 months. All the patients in this group were infused with RTX at $500 \mathrm{mg}$, then $100 \mathrm{mg}$ re-injected if the percentage of $\mathrm{CD} 19^{+} \mathrm{B}$ cells reached $1 \%$. This study was approved by the Chinese PLA General Hospital Ethical Review Board (IRB No. S2020042-02), and all patients provided written informed consent to participate in the study.

Descriptive statistical methods were utilized to report frequencies and distribution of the results and means \pm standard deviation (SD). For categorical data analysis, a chi-square test was used, and the means among continuous variables were adopted and tested for normal distribution (Shapiro-Wilk test). Parametric tests were utilized for continuous variables that were normally distributed, otherwise non-parametric tests were used. Non-parametric tests (Wilcoxon matched-pairs rank sum and Kruskal-Wallis test) were applied to compare 
Table 1 Clinical characteristics of the patients with NMOSD receiving rituximab, azathioprine, or mycophenolate mofetil treatment according to seropositive and seronegative AQP4-Ab status

\begin{tabular}{|c|c|c|c|c|c|c|c|}
\hline \multirow[b]{2}{*}{ AQP4-Ab } & \multicolumn{2}{|c|}{$\operatorname{RTX}(n=23)$} & \multicolumn{2}{|c|}{$\operatorname{AZA}(n=32)$} & \multicolumn{2}{|c|}{ MMF $(n=21)$} & \multirow[t]{2}{*}{$p$ value } \\
\hline & + & - & + & - & + & - & \\
\hline$n$ & 14 & 9 & 13 & 19 & 8 & 13 & \\
\hline $\begin{array}{l}\text { Age at onset, median, years } \\
\text { (range) }\end{array}$ & $\begin{array}{l}32.0 \\
\quad(23-36)\end{array}$ & $\begin{array}{l}29.0 \\
\quad(12-39)\end{array}$ & $\begin{array}{l}34.0 \\
\quad(33-50)\end{array}$ & $\begin{array}{l}37.0 \\
\quad(18-76)\end{array}$ & $\begin{array}{l}38.0 \\
\quad(12-50)\end{array}$ & $\begin{array}{l}37.0 \\
\quad(24-71)\end{array}$ & 0.79 \\
\hline Female gender, $n(\%)$ & $12(86 \%)$ & $8(89 \%)$ & $11(85 \%)$ & $14(74 \%)$ & $7(88 \%)$ & $10(77 \%)$ & 0.08 \\
\hline $\begin{array}{l}\text { Disease duration, median, years } \\
\text { (range) }\end{array}$ & $\begin{array}{l}12.4 \\
\quad(8-15)\end{array}$ & $\begin{array}{l}10.1 \\
\quad(8-12)\end{array}$ & $\begin{array}{l}11.9 \\
(7-13)\end{array}$ & $\begin{array}{l}10.8 \\
(6-15)\end{array}$ & $\begin{array}{l}11.5 \\
\quad(5-12)\end{array}$ & $\begin{array}{l}10.0 \\
\quad(8-13)\end{array}$ & 0.12 \\
\hline $\begin{array}{l}\text { Time since last relapse } \\
\text { (months) }\end{array}$ & $15.9(13.3)$ & $17.1(14.8)$ & $20.8(19.6)$ & $15.1(17.3)$ & $18.2(14.1)$ & $19.2(21.3)$ & 0.93 \\
\hline \multicolumn{8}{|l|}{ Clinical features } \\
\hline Optic neuritis, $n(\%)$ & $12(86 \%)$ & $6(67 \%)$ & $9(69 \%)$ & $15(79 \%)$ & $6(75 \%)$ & $10(77 \%)$ & 0.41 \\
\hline Myelitis, $n$ (\%) & $13(93 \%)$ & $7(78 \%)$ & $12(92 \%)$ & $16(84 \%)$ & $7(86 \%)$ & $12(92 \%)$ & 0.86 \\
\hline $\begin{array}{l}\text { Associated other autoimmune } \\
\text { disease } n(\%)\end{array}$ & $5(36 \%)$ & $4(44 \%)$ & $6(46 \%)$ & $4(21 \%)$ & $3(38 \%)$ & $2(20 \%)$ & 0.07 \\
\hline $\begin{array}{l}\text { Oral prednisolone dose before } \\
\text { immunosuppressant drugs } \\
\text { therapy (mean, mg/day) }\end{array}$ & $16.4 \pm 9.7$ & $12.9 \pm 8.7$ & $18.2 \pm 8.0$ & $10.6 \pm 6.7$ & $11.6 \pm 8.9$ & $12.5 \pm 7.2$ & 0.24 \\
\hline $\begin{array}{l}\text { Oral prednisolone dose after } \\
\text { immunosuppressant drugs } \\
\text { therapy (mean, mg/day) }\end{array}$ & $13.6 \pm 9.7$ & $16.1 \pm 8.6$ & $16.4 \pm 7.7$ & $11.4 \pm 7.3$ & $12.3 \pm 8.8$ & $11.6 \pm 8.1$ & 0.19 \\
\hline $\begin{array}{l}\text { Baseline EDSS before } \\
\text { treatment, median (range) }\end{array}$ & $\begin{array}{l}4.0 \\
(3.5-5.5)\end{array}$ & $\begin{array}{l}3.0^{\mathrm{a} 1} \\
\quad(1.5-4.5)\end{array}$ & $3.5(2-4.5)$ & $\begin{array}{l}3.0^{\mathrm{a} 3} \\
\quad(2-5.5)\end{array}$ & $\begin{array}{l}3.0^{\mathrm{b} 1} \\
(2.0-4.0)\end{array}$ & $\begin{array}{l}3.5^{95, \mathrm{~b} 3} \\
(2.0-4.0)\end{array}$ & 0.23 \\
\hline $\begin{array}{l}\text { Baseline ARR before } \\
\text { treatment, median (range) }\end{array}$ & $1.0(0.2-3)$ & $\begin{array}{l}0.3^{\mathrm{a} 2} \\
(0.2-1.5)\end{array}$ & $\begin{array}{l}0.8 \\
\quad(0.3-1.3)\end{array}$ & $\begin{array}{l}0.9^{a 4} \\
(0.5-1.3)\end{array}$ & $\begin{array}{l}0.85^{\mathrm{b} 2} \\
(0.5-1.5)\end{array}$ & $\begin{array}{l}0.9^{\mathrm{a}, \mathrm{b}, \mathrm{b}} \\
(0.4-1.5)\end{array}$ & 0.18 \\
\hline
\end{tabular}

Patients with neuromyelitis optica spectrum disorders (NMOSD) who tested seropositive or seronegative for the aquaporin4 antibody ( $\mathrm{AQP} 4-\mathrm{Ab}$ ) were divided into three groups based on immunosuppressant therapy initiation. There was no significant difference of baseline characteristics among these patients who received RTX, AZA, and MMF therapies $(p>0.05)$. Moreover, there was no significant difference in oral prednisone dose before and after RTX, AZA, and MMF immunosuppressant therapy among patients with NMOSD with seropositive and seronegative AQP4-Ab $(p>0.05)$. The titer of antibody more than or equal to $1: 100$ was recognized as a seropositive status

$R T X$ rituximab, $A Z A$ azathioprine, $M M F$ mycophenolate mofetil, $A Q P 4$ aquaporin-4, EDSS Expanded Disability Status Scale, $A R R$ annualized relapse rate

${ }^{a} P$ value was calculated between seropositive AQP4 antibody and seronegative AQP4 antibody in RTX, AZA, and MMF groups, respectively $(P$ a1, a2, a3, a4, a5, a6 $>0.05)$

${ }^{\mathrm{b}} P$ value was calculated among the three groups with RTX, AZA, and MMF, respectively, in seropositive AQP4 antibody and seronegative AQP4 antibody groups ( $P$ b1, b2, b3, b4 > 0.05) 


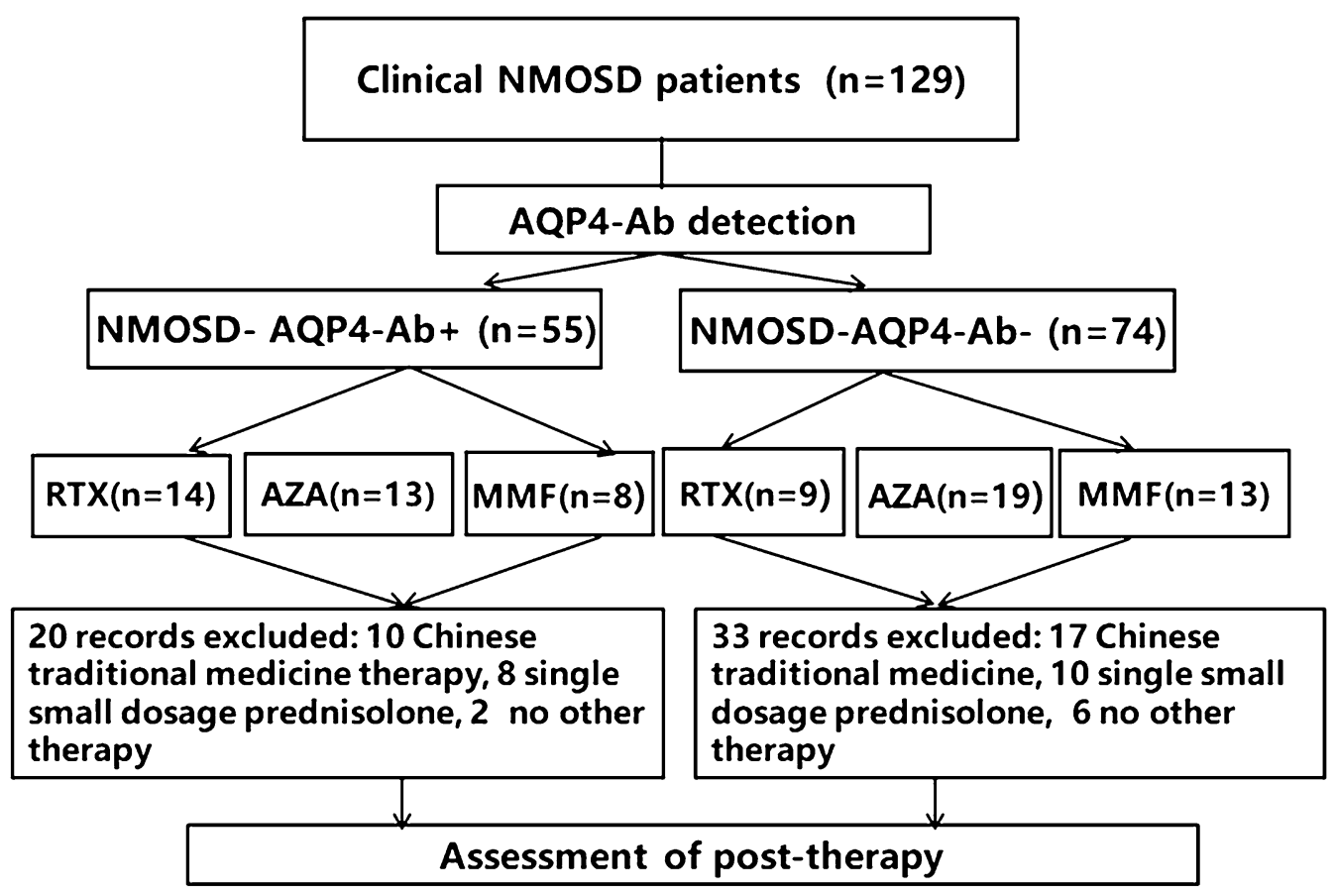

Fig. 1 Flowchart of the study

pre-treatment and post-treatment relapse rates and Expanded Disability Status Scale (EDSS) scores. The EDSS, performed by a neurologist, was also registered for each patient. A Kaplan-Meier survival curve was prepared on the basis of estimates. SPSS software version 14.0 was used to perform all statistical analyses. $P$ values less than 0.05 were considered statistically significant.

\section{RESULTS}

\section{Baseline Clinical Characteristics of Patients with NMOSD}

The baseline clinical characteristics of the patients with NMOSD are shown in Table 1. A total of 129 patients were enrolled in the study with 55 seropositive and 74 seronegative AQP4$\mathrm{Ab}$, including 23 patients in the RTX group with 14 seropositive and nine seronegative AQP4-Ab, 32 patients in the AZA group with 13 seropositive and 19 seronegative AQP4-Ab, 21 patients in the MMF group with eight seropositive and 13 seronegative AQP4-Ab (Fig. 1, Table 1). The median onset age was 32 years old (range 23-36), with 12 women (86\%), and the median disease duration was 12.4 years (range 8-15) in the RTX group with seropositive AQP4-Ab. Optic neuritis $(n=12,86 \%)$ and acute myelitis ( $n=13,93 \%)$ were the most common clinical manifestations. Five (36\%) patients were associated with other autoimmune diseases. The median EDSS score was 4.0 (3.5-5.5) before treatment with RTX.

The median onset age was 34 years old (range 33-50), with 11 women (85\%), and the median disease duration was 11.9 years (range 7-13) in the AZA group with seropositive AQP4Ab. Optic neuritis $(n=9,69 \%)$ and acute myelitis ( $n=12,92 \%)$ were the most common clinical manifestations. Six (46\%) patients were associated with other autoimmune diseases. The median EDSS score was 3.5 (2-4.5) before treatment with AZA.

The median onset age was 38 years old (range 12-50), with seven female patients $(88 \%)$, and the median disease duration was 11.5 years (range 5-12) in the MMF group with seropositive AQP4-Ab. Optic neuritis $(n=6$, $75 \%)$ and acute myelitis $(n=7,86 \%)$ were the 
most common clinical manifestations. Three (38\%) patients were associated with other autoimmune diseases. The median EDSS score was 3.0 (2.0-4.0) before treatment with MMF.

On the basis of the aforementioned analysis results, there was no significant difference in baseline characteristics among these patients who received RTX, AZA, and MMF therapy $(P>0.05)$. In addition, all patients were taking prednisolone at enrollment, and there was no significant different oral prednisone dose before and after RTX, AZA, and MMF immunosuppressant therapy among patients with NMOSD with seropositive and seronegative AQP4-Ab $(P>0.05)$. Nevertheless, the basal characteristics were lacking epidemiological and radiological variables.

\section{Effectiveness of Rituximab, Azathioprine, and Mycophenolate Mofetil Treatment in Patients with NMOSD}

In the group treated with RTX, the EDSS scores decreased from a pre-treatment value of 4.0 (3.5-5.5) to a post-treatment value of 4.0 (3.5-5.5) at the last follow-up (median 90 months). The annual relapse rates (ARR) before treatment were defined as the ARR calculated before the first injection. ARR after treatment was defined as the ARR calculated between the first injection and the last followup. As a result, the median post-treatment ARR was $0.7(0.1-2)$, compared with the pre-treatment value of $1.0(0.2-3)$ in the RTX group with seropositive AQP4-Ab $(P<0.05$, Wilcoxon matched-pairs rank sum test). Meanwhile, the EDSS scores decreased from a pre-treatment score of $3.0(1.5-4.5)$ to a post-treatment score of 2.5 (1.5-3.5) at the last follow-up (median 90 months). As a result, the median post-treatment ARR was $0.2(0.1-0.6)$, compared with the pre-treatment rate $0.3(0.2-1.5)$ in the RTX group with seronegative AQP4-Ab $(P<0.05$, Wilcoxon matched-pairs rank sum test).

In the group treated with AZA, the EDSS scores decreased from a pre-treatment value of $2.5(2.0-3.5)$ to a pre-treatment score of 3.5 (2.0-4.5). The median post-treatment ARR was $0.3(0.1-1.0)$, compared with the pre-treatment rate of $0.8(0.3-1.3)$ in the AZA group with seropositive AQP4-Ab $(P<0.05$, Wilcoxon matched-pairs rank sum test). Meanwhile, the EDSS scores decreased from a pre-treatment score of $3.0(2.0-5.5)$ to a post-treatment score of $2.5(1.0-4.0)$ at the last follow-up (median 90 months). The median post-treatment ARR was $0.5(0.2-1.0)$, compared with the pre-treatment rate of $0.9(0.5-1.3)$ in the AZA group with seronegative AQP4-Ab $(P<0.05$, Wilcoxon matched-pairs rank sum test).

In the group treated with MMF, the EDSS scores decreased from a pre-treatment score of $3.0(2.0-4.0)$ to a post-treatment score of 2.0 (1.5-2.5). The median post-treatment ARR was 0.35 (0.1-1.0), compared with the pre-treatment rate of $0.85(0.5-1.5)$ in the MMF group with seropositive AQP4-Ab $(P<0.05$, Wilcoxon matched-pairs rank sum test). Meanwhile, the EDSS scores decreased from a pre-treatment score of $3.5(2.0-4.0)$ to a post-treatment score of 2.0 (1.5-3.5) at the last follow-up (median 90 months). The median post-treatment ARR was $0.4(0.2-0.9)$, compared with the pre-treatment rate of $0.9(0.4-1.5)$ in the MMF group with seronegative AQP4-Ab $(P<0.05$, Wilcoxon matched-pairs rank sum test). The clinical characteristics are summarized in Tables 1 and 2 .

\section{Long-Term Follow-up in NMOSD}

Medical record reviews and telephone followups were conducted for the 76 patients with NMO/NMOSD receiving RTX, AZA, and MMF treatment from January 2008 to November 2018. Medical record reviews in these patients provided the post-treatment follow-up data. The median follow-up of all patients was 90 (68-104) months. There were 76 patients with NMOSD treated with these three drugs at the last review; owing to the low incidence of this disorder and dropout, patients received other therapy. In the last review, there were 23,32 , and 21 patients treated with RTX, AZA, and $\mathrm{MMF}$, respectively, with a median duration of 18 (12-49), 25 (18-51), and 22 (12-48) months treatment. EDSS and ARR after initiation of RTX, AZA, and MMF until the last follow-up 
Table 2 Effectiveness of Immunosuppressive treatment in patients with NMOSD

\begin{tabular}{|c|c|c|c|c|c|c|}
\hline \multirow[b]{2}{*}{ AQP4-Ab } & \multicolumn{2}{|c|}{$\operatorname{RTX}(n=23)$} & \multicolumn{2}{|c|}{$\operatorname{AZA}(n=32)$} & \multicolumn{2}{|c|}{$\operatorname{MMF}(n=21)$} \\
\hline & + & - & + & - & + & - \\
\hline$n$ & 14 & 9 & 13 & 19 & 8 & 13 \\
\hline $\begin{array}{l}\text { EDSS before treatment, } \\
\text { median (range) }\end{array}$ & $\begin{array}{l}4.0 \\
(3.5-5.5)\end{array}$ & $\begin{array}{l}3.0^{\mathrm{a} 1} \\
\quad(1.5-4.5)\end{array}$ & $3.5(2-4.5)$ & $3.0^{\mathrm{a} 5}(2-5.5)$ & $3.0(2.0-4.0)$ & $3.5^{\mathrm{a9}}(2.0-4.0)$ \\
\hline $\begin{array}{l}\text { ARR before treatment, median } \\
\text { (range) }\end{array}$ & $1.0(0.2-3)$ & $\begin{array}{l}0.3^{\mathrm{a} 2} \\
\quad(0.2-1.5)\end{array}$ & $\begin{array}{l}0.8 \\
\quad(0.3-1.3)\end{array}$ & $\begin{array}{l}0.9^{\mathrm{a} 6} \\
\quad(0.5-1.3)\end{array}$ & $\begin{array}{l}0.85 \\
\quad(0.5-1.5)\end{array}$ & $\begin{array}{l}0.9^{\mathrm{a} 10} \\
\quad(0.4-1.5)\end{array}$ \\
\hline $\begin{array}{l}\text { EDSS after treatment, median } \\
\quad \text { (range) }\end{array}$ & $\begin{array}{l}2.75^{\mathrm{b} 1} \\
\quad(2.5-3)\end{array}$ & $\begin{array}{l}2.5^{\mathrm{a}, \mathrm{b} 3} \\
\quad(1.5-3.5)\end{array}$ & $\begin{array}{l}2.5^{\mathrm{b} 5} \\
\quad(2-3.5)\end{array}$ & $\begin{array}{l}2.5^{\mathrm{a}, \mathrm{b} 7} \\
\quad(1.0-4.0)\end{array}$ & $\begin{array}{l}2.0^{\mathrm{b} 9} \\
(1.5-2.5)\end{array}$ & $\begin{array}{l}2.0^{\mathrm{a} 11, \mathrm{~b} 11} \\
\quad(1.5-3.5)\end{array}$ \\
\hline $\begin{array}{l}\text { ARR after treatment, median } \\
\text { (range) }\end{array}$ & $\begin{array}{l}0.7^{\mathrm{b} 2} \\
(0.1-2)\end{array}$ & $\begin{array}{l}0.2^{\mathrm{a}, \mathrm{b} 4} \\
(0.1-0.6)\end{array}$ & $\begin{array}{l}0.3^{\mathrm{b} 6} \\
(0.1-1.0)\end{array}$ & $\begin{array}{l}0.5^{\mathrm{a}, \mathrm{b} 8} \\
\quad(0.2-1.0)\end{array}$ & $\begin{array}{l}0.35^{\mathrm{b} 10} \\
(0.1-1.0)\end{array}$ & $\begin{array}{l}0.4^{\mathrm{a} 12, \mathrm{~b} 12} \\
(0.2-0.9)\end{array}$ \\
\hline
\end{tabular}

An antibody titer of more than or equal to 1:100 was recognized as a seropositive status

$R T X$ rituximab, $A Z A$ azathioprine, $M M F$ mycophenolate mofetil, EDSS Expanded Disability Status Scale, $A R R$ annualized relapse rate

${ }^{\mathrm{a}} P$ value was calculated between seropositive AQP4 antibody and seronegative AQP4 antibody in RTX $(p=0.001)$, AZA, and MMF groups, respectively. $P$ a $1=0.001, P$ a2 $=0.001, P$ a3 $=0.085, P$ a4 $=0.088 ; P$ a5 $=0.963, P$ a6 $=0.268, P$ a7 $=0.659, P$ a8 $=0.357 ; P$ a9 $=0.651, P$ a10 $=0.697, P$ a1 $1=0.581, P$ a $12=0.645$

${ }^{\mathrm{b}} P$ value was calculated between pre-therapy and post-therapy with RTX, AZA, and MMF, respectively, in seropositive AQP4 antibody and seronegative AQP4 antibody groups. $P$ b1 $<0.001, P$ b2 $=0.001, P$ b3 $=0.002, P$ b4 $<0.001$; $P$ b5 $=0.003, P$ b6 $=0.004, P$ b7 $<0.001, P$ b8 $<0.001 ; P$ b9 $=0.002, P$ b10 $=0.002, P$ b11 $<0.001, P$ b12 $<0.001$

were analyzed. The EDSS reduction ratios were $0.70 \pm 0.23,0.37 \pm 0.19$, and $0.2 \pm 0.13$, and ARR reduction ratios were $0.27 \pm 0.11$ (RTX), $0.16 \pm 0.05$ (AZA), and $0.09 \pm 0.03$ (MMF), respectively. The changes of EDSS and relapses rates were shown before and after treatment. There was a significant reduction compared with the pre-treatment values in EDSS and ARR $(P<0.05)$ (Table 2, Fig. 2, Supplementary Figs. 1 and 2). However, there was no statistically significant difference between the post-treatment and pre-treatment changes of EDSS and ARR in RTX, AZA, and MMF groups $(P>0.05)$ (Table 2 , Fig. 3).

\section{Adverse Effects}

Adverse effects were reported in a few patients: 1 (7.7\%) patient treated with RTX had an infection (gingivitis), and $2(6.3 \%)$ had low counts of white blood cells; only one patient $\left(\mathrm{CD} 19^{+}\right.$count $\left.0.01 \%\right)$ had rebound (severe high paraplegia) without routine infusion low-dose RTX. Furthermore, $87 \%(20 / 23)$ of patients reported no adverse effects following RTX therapy (very low dose used).

Two (6.3\%) patients treated with AZA had low counts of white blood cells and three (9.3\%) experienced liver dysfunction; one (4.8\%) patient treated with MMF had low counts of white blood cells and two (9.5\%) experienced liver dysfunction. No case had hematological malignancy during the 90 months follow-up. Furthermore, there were no serious side effecs reported in the three groups (Table 3).

\section{Kaplan-Meier Survival Analysis}

The Kaplan-Meier survival estimates indicated significant differences in relapse rates among the subjects treated with RTX, AZA, and MMF $(P<0.05)$ (Fig. 4). Namely, RTX-treated patients were more likely to be relapse-free after long- 

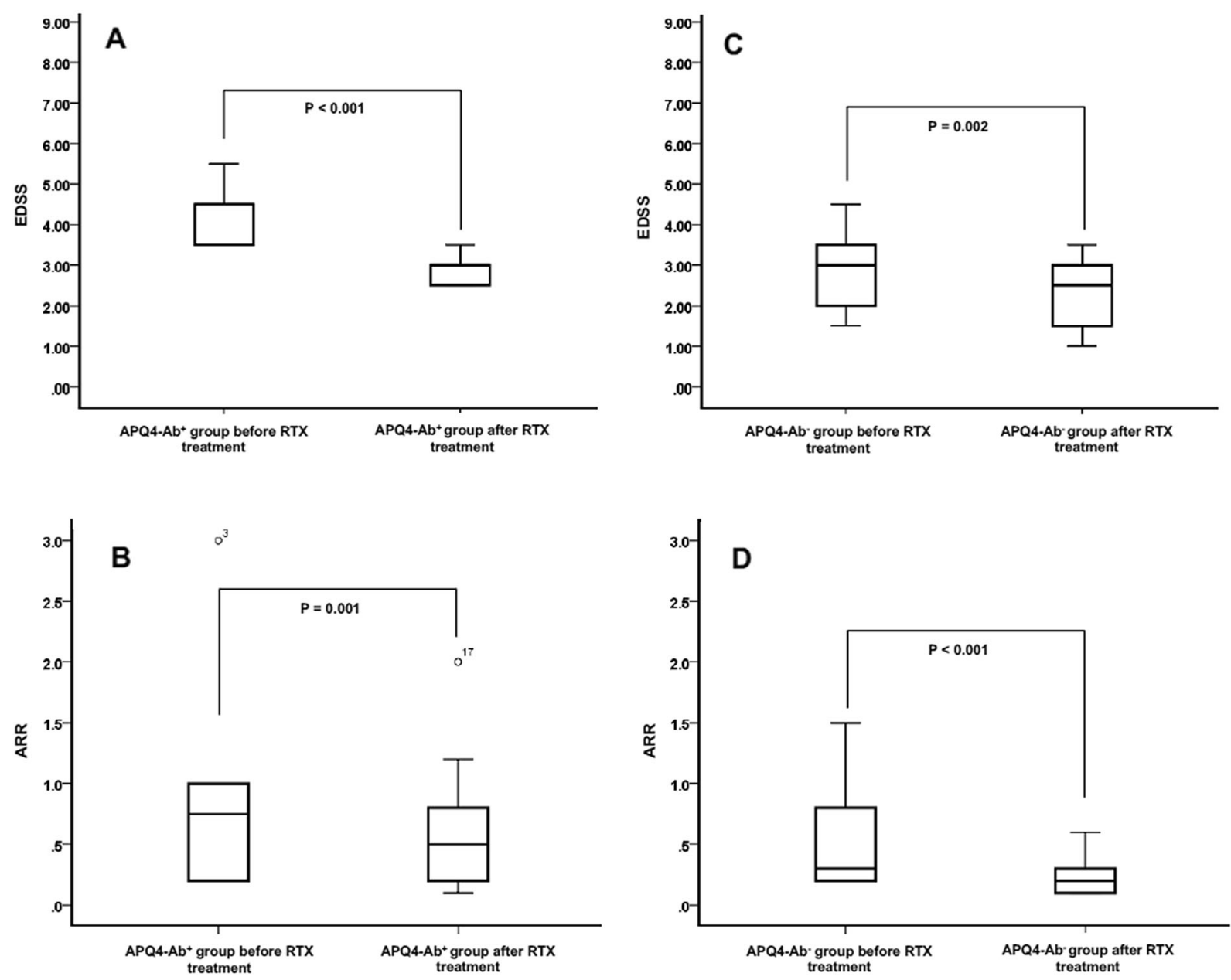

Fig. 2 Changes of EDSS (a, c) and ARR (b, d) after RTX treatment either in seropositive anti-AQP4 antibody patients $(\mathbf{a}, \mathbf{b})$ or seronegative anti-AQP4 antibody patients $(\mathbf{c}, \mathbf{d})$. There were significant differences in the EDSS $(\mathbf{a}, \mathbf{c})$ and $\operatorname{ARR}(\mathbf{b}, \mathbf{d})(P<0.05)$. EDSS Expanded

term follow-up than those who received AZA or MMF therapy.

\section{DISCUSSION}

In this study, Chinese patients with NMOSD and either AQP4-Ab seropositive or seronegative status were enrolled, and their immunosuppressant therapies were studied. As the latest diagnostic criteria for NMOSD in 2015 included seropositive AQP4-Ab status [26], seropositive AQP4-Ab patients with NMO and high disease activity could more easily relapse and should be

Disability Status Scale, ARR annualized relapses rate, RTX rituximab

carefully followed and managed. Thus, it is essential to assess the long-term effects and safety of NMO with immunosuppressant treatment between AQP4-Ab seropositive and negative status.

Previous studies have illustrated that the estimated risk of relapsing was 30\% after 5 years without immunosuppressant therapy and 50\% after 10 years [6]. Further, our research team had determined that $12.1 \%$ of patients with initial optical neuritis (ON) attacks could progress to NMO within 5 years [28]. Our current study involved a median of 90 months' follow-up of patients with NMO to investigate the associated 


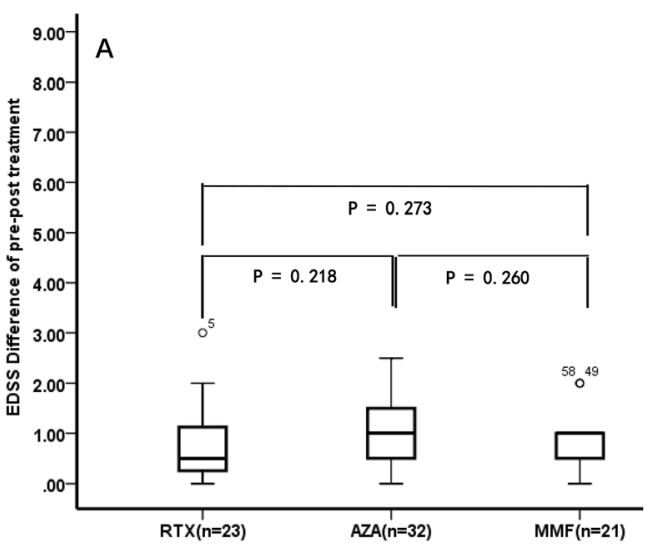

Fig. 3 Changes of EDSS (a) and ARR (b) after RTX, AZA, and MMF treatment versus pre-treatment. There was no statistically significant difference among the three

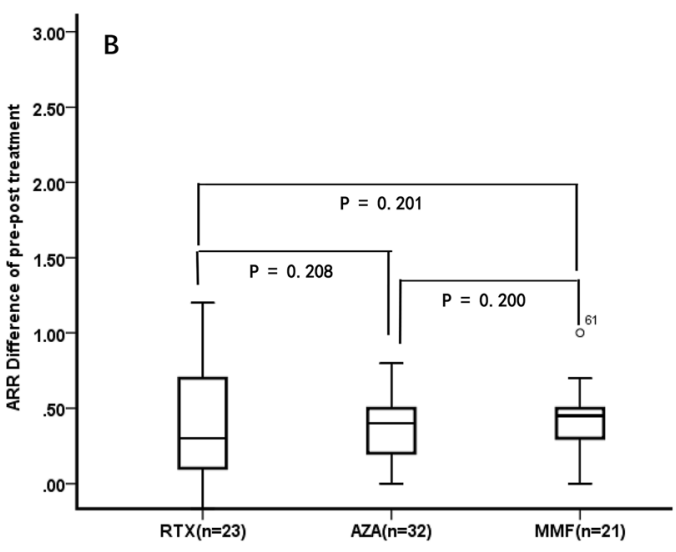

groups $(P>0.05)$. EDSS Expanded Disability Status Scale, ARR annualized relapses rate, RTX rituximab, AZA azathioprine, MMF mycophenolate mofetil

Table 3 Side effects of immunosuppressive treatment in patients with NMOSD during long-term follow-up

\begin{tabular}{|c|c|c|c|c|c|c|}
\hline \multirow[b]{2}{*}{ AQP4-Ab } & \multicolumn{2}{|l|}{$\operatorname{RTX}(n=23)$} & \multicolumn{2}{|c|}{$\operatorname{AZA}(n=32)$} & \multicolumn{2}{|c|}{ MMF $(n=21)$} \\
\hline & + & - & + & - & + & - \\
\hline$n$ & 14 & 9 & 13 & 19 & 8 & 13 \\
\hline Adverse events frequently reported & 1 & 2 & 3 & 2 & 2 & 1 \\
\hline Rebound of relapse after withdrawal & 1 & 0 & 0 & 0 & 0 & 0 \\
\hline Infection & 1 (gingivitis, $7.7 \%$ ) & 0 & 0 & 0 & 0 & 0 \\
\hline Blood routine (LWBC) & 0 & 0 & $2(6.3 \%)$ & 0 & $1(4.8 \%)$ & 0 \\
\hline Liver dysfunction & 0 & 0 & 0 & 0 & $2(9.5 \%)$ & 0 \\
\hline Hematological malignancy & 0 & 0 & 0 & 0 & 0 & 0 \\
\hline
\end{tabular}

An antibody titer of more than or equal to $1: 100$ was recognized as a seropositive status $R T X$ rituximab, $A Z A$ azathioprine, $M M F$ mycophenolate mofetil, EDSS Expanded Disability Status Scale, ARR annualized relapse rate, $L W B C$ low white blood cell counts

clinical characteristics and long-term prognosis. In this study, the tolerance of RTX was good in most cases and had acceptable safety results, and it effectively decreased disability scores (EDSS) and relapse rates (ARR). Moreover, there were significant differences in the post-treatment and pre-treatment changes of EDSS and ARR with RTX either in the $\mathrm{AQP4}^{+}$or AQP4 ${ }^{-}$antibody group. These results support that RTX, a drug efficiently used in demyelinating spectrum diseases [22], could also treat patients with NMOSD. The present retrospective research summarized the treatment experience at our clinic with these drugs. Low-dose RTX, AZA, and MMF therapies were all effective in reducing relapse and improving or stabilizing the disabilities, regardless of seropositive or negative AQP4-Ab status, as shown by the improvements in the EDSS and ARR. However, there was no statistically significant difference in the post-treatment and pretreatment changes of EDSS and ARR among RTX, AZA, and MMF groups. 


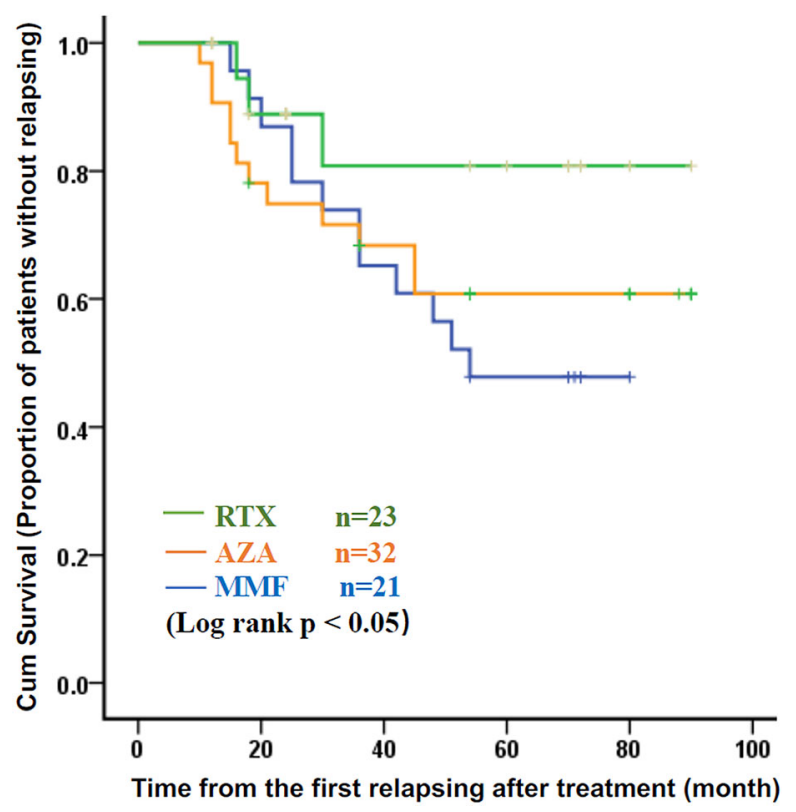

Fig. 4 Kaplan-Meier survival estimates pertaining to probabilities of being without relapse after RTX (rituximab, top line), AZA (azathioprine, middle line), and MMF (mycophenolate mofetil, bottom line) treatment. Kaplan-Meier survival estimates indicated significant differences in relapse rates between RTX- and AZAtreated subjects, and in relapse rates between RTX- and MMF-treated subjects, but not significant difference in relapse rates between MMF- and AZA-treated subjects. Namely, RTX-treated patients were more likely to be relapse-free after long-term follow-up than those who received AZA or MMF therapy $(\log \operatorname{rank} p<0.05)$

As a monoclonal antibody that targets $\mathrm{CD}^{2} \mathrm{O}^{+} \mathrm{B}$ cells, rituximab has been used to prevent NMO attacks [16]. The precise mechanism of the effect of rituximab in people with NMO has not been established, though the depletion of B cells is recognized as an effective target [15]. RTX treatment causes depletion of $\mathrm{CD}_{2} \mathrm{O}^{+}$and $\mathrm{CD} 19^{+} \mathrm{B}$ cells. In a recent randomized placebocontrolled clinical trial, rituximab completely prevented relapses in all patients with $\mathrm{NMO}$ and seronegative AQP4-Ab status [15]. In our study, the improvement of EDSS and the rate of reduction in risk of relapse were apparent in both seropositive and seronegative patients.

RTX has a potential beneficial effect; however, it requires intravenous infusions and is associated with infection risk that may limit its use, especially in treating NMO as a first-class agent. According to our study, although no significant difference was observed among the RTX, MMF, and AZA groups in EDSS and ARR values at pre-post treatment, the Kaplan-Meier survival estimates indicated there were significant differences in relapse rates among the subjects treated with RTX, AZA, and MMF, and RTX-treated patients were more likely to be relapse-free after long-term follow-up than those who received AZA or MMF therapy. For this reason, on the basis of our study, AZA or MMF may also produce adverse effects rather than serious side effects. In total, 87\% (20/23) of patients reported no adverse effects following RTX therapy (low dose used). In most patients, the use of RTX was limited because of the high cost and the need to monitor CD19 ${ }^{+}$lymphocytes with flow cytometry analysis [7, 18, 25].

Moreover, less than $6.3 \%$ of our patients experienced low white blood cell counts, and less than $7.7 \%$ experienced infection (such as gingivitis). Therefore, patients and physicians need to be aware of the long-term risks of hematological malignancies. Although we have not found hematological malignancy cases, the awareness of the risk should be emphasized.

We also note the limitations of our study such as the small sample size, the lack of a randomized placebo-controlled design, and lack of the basal epidemiological and radiological characters. Also, concomitant treatments may preclude a definitive evaluation of the efficacy of RTX, AZA, and MMF in NMO relapse prevention [29]. In addition, a washout period to eliminate the influence of prior therapies was absent. Finally, there were more seronegative patients with NMOSD than seropositive ones in this cohort, and the presence of other independent disease groups in the seronegative NMOSD group may substantially affect the results.

\section{CONCLUSIONS}

Our findings suggest that RTX, MMF, and AZA are all effective in patients with NMOSD both in seropositive and seronegative AQP4-Ab status. Specifically, RTX-treated patients were more likely to be relapse-free after long-term follow- 
up. Thus, despite the mentioned limitations, this study provided evidence supporting the use of RTX, AZA, and MMF to prevent NMO attacks. RTX, AZA, and MMF seemed to be effective, even with or without oral prednisone administration; however, the effectiveness, cost, and adverse effects associated with these treatments, and the urgency to achieve immediate immunosuppression, could practically affect the choice of treatment. Nevertheless, RTX should be recommended owing to its long-term efficacy and safety. Further research is ongoing that is based on part of these individual cases generated by our clinical database.

\section{ACKNOWLEDGEMENTS}

This study was carried out with the adequate understanding and consent of the patients. Therefore we want to thank all participants and their families. In addition, we thank Prof. Yanhong Tai (Department of Neuropathology, The Five Medical Center of Chinese PLA General Hospital, Beijing, China) for statistical assistance with the article. We also thank Prof. Wei Chen for editing assistance of the revision.

Funding. This research received a grant from a healthcare funding agency in the military scientific research institute (Military health-care project, No. 18BJZ34). The journal's Rapid Service Fee was funded by the authors.

Authorship. All named authors meet the International Committee of Medical Journal Editors (ICMJE) criteria for authorship for this article, take responsibility for the integrity of the work as a whole, and give their approval for this version to be published.

Authors' Contributions. Data analysis: Yang Yang, Jiarui Yao, Lifeng Chen. Investigation: Yang Yang, Jiarui Yao, Tianyu Jiang, Lei Wu. Methodology: Dehui Huang and Weiping Wu. Formal design: Weiping $\mathrm{Wu}$ and Zhenfu Wang. Writing-review and editing: Yang Yang and Jiarui Yao. Clinical data collection and curation: Xiaoqing Su, Dongmei Li, Lina Han.
Disclosures. The authors (Yang Yang, Lifeng Chen, Lei $\mathrm{Wu}$, Jiarui Yao, Na Wang, Xiaoqing $\mathrm{Su}$, Dongmei Li, Lina Han, Weiping Wu, Dehui Huang, Tianyu Jiang, Zhenfu Wang) have nothing to disclose, and no conflict of interest to report.

Compliance with Ethics Guidelines. This study was approved by the Ethics Committee in General Hospital of Chinese PLA (IRB No. S2020-042-02).

Data Availability. All data generated or analyzed during this study are included in this published article and supplementary information files.

Open Access. This article is licensed under a Creative Commons Attribution-NonCommercial 4.0 International License, which permits any non-commercial use, sharing, adaptation, distribution and reproduction in any medium or format, as long as you give appropriate credit to the original author(s) and the source, provide a link to the Creative Commons licence, and indicate if changes were made. The images or other third party material in this article are included in the article's Creative Commons licence, unless indicated otherwise in a credit line to the material. If material is not included in the article's Creative Commons licence and your intended use is not permitted by statutory regulation or exceeds the permitted use, you will need to obtain permission directly from the copyright holder. To view a copy of this licence, visit http://creativecommons.org/licenses/bync/4.0/.

\section{REFERENCES}

1. Hinson SR, Pittock SJ, Lucchinetti CF, et al. Pathogenic potential of IgG binding to water channel extracellular domain in neuromyelitis optica. Neurology. 2007;69:2221-31.

2. Lennon VA, Wingerchuk DM, Kryzer TJ, et al. A serum autoantibody marker of neuromyelitis optica: distinction from multiple sclerosis. Lancet. 2004;364:2106-12. 
3. Weinshenker BG, Wingerchuk DM, Vukusic S, et al. Neuromyelitis optica IgG predicts relapse after longitudinally extensive transverse myelitis. Ann Neurol. 2006;59:566-9.

4. Matiello M, Lennon VA, Jacob A, et al. NMO-IgG predicts the outcome of recurrent optic neuritis. Neurology. 2008;70:2197-200.

5. Matiello M, Jacob A, Wingerchuk DM, Weinshenker BG. Neuromyelitis optica. Curr Opin Neurol. 2007;20:255-60.

6. Mandler RN, Ahmed W, Dencoff JE. Devic's neuromyelitis optica: a prospective study of seven patients treated with prednisone and azathioprine. Neurology. 1998;51:1219-20.

7. Jacob A, Matiello M, Weinshenker BG, et al. Treatment of neuromyelitis optica with mycophenolate mofetil. Arch Neurol. 2009;66:1128-33.

8. Huh SY, Kim SH, Hyun JW, et al. Mycophenolate mofetil in the treatment of neuromyelitis optica spectrum disorder. JAMA Neurol. 2014;71:1372-8.

9. Huang Q, Wang J, Zhou Y, et al. Low-dose mycophenolate mofetil for treatment of neuromyelitis optica spectrum disorders: a prospective multicenter study in South China. Front Immunol. 2018;9:2066.

10. Chen H, Qiu W, Zhang Q, et al. Comparisons of the efficacy and tolerability of mycophenolate mofetil and azathioprine as treatments for neuromyelitis optica and neuromyelitis optica spectrum disorder. Eur J Neurol. 2017;24:219-26.

11. Watanabe S, Misu T, Miyazawa I, et al. Low-dose corticosteroids reduce relapses in neuromyelitis optica: a retrospective analysis. Mult Scler. 2007;13: 968-74.

12. Weinstock-Guttman B, Ramanathan M, Lincoff $N$, et al. Study of mitoxantrone for the treatment of recurrent neuromyelitis optica (Devic disease). Arch Neurol. 2006;63:957-63.

13. Stellmann JP, Krumbholz M, Friede $\mathrm{T}$, et al. Immunotherapies in neuromyelitis optica spectrum disorder efficacy and predictors of response. J Neurol Neurosurg Psychiatry. 2017;88:639-47.

14. Zhang M, Zhang C, Bai P, Xue H, Wang G. Effectiveness of low dose of rituximab compared with azathioprine in Chinese patients with neuromyelitis optica: an over 2-year follow-up study. Acta Neurol Belg. 2017;117:695-702.

15. Tahara M, Oeda T, Okada K, et al. Safety and efficacy of rituximab in neuromyelitis optica spectrum disorders (RIN-1 study): a multicentre, randomised, double-blind, placebo-controlled trial. Lancet Neurol. 2020;19:298-306.

16. Shaygannejad V, Fayyazi E, Badihian S, et al. Longterm tolerability, safety and efficacy of rituximab in neuromyelitis optica spectrum disorder: a prospective study. J Neurol. 2019;266:642-50.

17. Pellkofer HL, Krumbholz M, Berthele A, et al. Longterm follow-up of patients with neuromyelitis optica after repeated therapy with rituximab. Neurology. 2011;76:1310-5.

18. Lu Q, Luo J, Hao H, et al. A long-term follow-up of rituximab treatment in 20 Chinese patients with neuromyelitis optica spectrum disorders. Mult Scler Relat Disord. 2020;40:101933.

19. Kim SH, Kim Y, Kim G, et al. Less frequent rituximab retreatment maintains remission of neuromyelitis optica spectrum disorder, following long-term rituximab treatment. J Neurol Neurosurg Psychiatry. 2019;90:486-7.

20. Kim SH, Huh SY, Lee SJ, Joung A, Kim HJ. A 5-year follow-up of rituximab treatment in patients with neuromyelitis optica spectrum disorder. JAMA Neurol. 2013;70:1110-7.

21. Jacob A, Weinshenker BG, Violich I, et al. Treatment of neuromyelitis optica with rituximab: retrospective analysis of 25 patients. Arch Neurol. 2008;65:1443-8.

22. D'Amico E, Zanghi A, Chisari CG, et al. Effectiveness and safety of rituximab in demyelinating diseases spectrum: an Italian experience. Mult Scler Relat Disord. 2019;27:324-6.

23. Cree BA, Lamb S, Morgan K, Chen A, Waubant E, Genain C. An open label study of the effects of rituximab in neuromyelitis optica. Neurology. 2005;64:1270-2.

24. Cabre P, Mejdoubi M, Jeannin S, et al. Treatment of neuromyelitis optica with rituximab: a 2-year prospective multicenter study. J Neurol. 2018;265: 917-25.

25. Grillo-Lopez AJ. Rituximab: an insider's historical perspective. Semin Oncol. 2000;27:9-16.

26. Wingerchuk DM, Banwell B, Bennett JL, et al. International consensus diagnostic criteria for neuromyelitis optica spectrum disorders. Neurology. 2015;85:177-89.

27. Mader S, Lutterotti A, Di Pauli F, et al. Patterns of antibody binding to aquaporin-4 isoforms in neuromyelitis optica. PLoS One. 2010;5:e104455. 
28. Zhou H, Zhao S, Yin D, et al. Optic neuritis: a 5-year follow-up study of Chinese patients based on aquaporin-4 antibody status and ages. J Neurol. 2016;263:1382-9.
29. Morton V, Torgerson DJ. Effect of regression to the mean on decision making in health care. BMJ. 2003;326:1083-4. 